\title{
Volumineux Mélanome Malin De La Conjonctive Multi Récidivant À Propos d'un Cas
}

\author{
Abba Kaka. H. Y \\ Ali.M H \\ Service Ophtalmologique Hôpital National De Niamey \\ Boubacar. $M$ \\ Service Ophtalmologique Hopital National Lamordé, Niamey \\ Moussa. F \\ Service Ophtalmologique Hôpital National De Niamey \\ Amza. A \\ Service Ophtalmologique Hopital National Lamordé, Niamey
}

\begin{abstract}
Melanoma of the conjunctiva is a tumor that develops from the conjunctival melenocytes. It often reoccurs even after several surgical removals. The authors present the case of a 45 years old woman who was at first seen in our outpatient clinic with a spitz naevus of the conjunctiva of the right eye since more than 20 years ago. This naevus which was excised twice and then the eye enucleated underwent malignant transformation into a voluminous multi-recidivant malignant melanoma weighing after surgical removal $350 \mathrm{~g}$ and measures $6 \times 12 \times 5 \mathrm{~cm}$.
\end{abstract}

Keywords: Melanoma, Conjunctival spitz naevus, metastasis, Niger

\section{Résumé}

Le mélanome de la conjonctive est une tumeur des mélanocytes conjonctivaux. Cette tumeur maligne donne parfois des récidives. Les auteurs rapportent le cas d'une patiente de 45 ans qui a été suivie de manière intermittente dans le service d'ophtalmologie et dans d'autres centres non spécialisés, pour un naevus de spitz conjonctival de l'œil droit. En 20 ans d'évolution et après deux exérèses et une énucléation de l'œil droit, cette néoformation a dégénéré en une tumeur volumineuse qui après ablation pesait $350 \mathrm{~g}$ et mesurait $6 \times 12 \times 5 \mathrm{~cm}$. L'examen anatomopathologique de cette tumeur a confirmé le diagnostic de mélanome de l'œil droit.

Mots clés: mélanome, naevus de spitz conjonctival, métastase, Niger 


\section{Introduction}

Le mélanome de la conjonctive est une tumeur rare des mélanocytes conjonctivaux qui se développe aux dépens des mélanocytes issus du neuroectoderme dans la couche basale de la conjonctive. Ils représentent seulement $2 \%$ des tumeurs malignes de l'œil. C'est une tumeur qui donne parfois des récidives qui peuvent se situer sur le site de la tumeur initiale, ou à distance, du fait de la migration de cellules tumorales dans les larmes (Desjardins L 1999). Le mélanome a un pouvoir métastatique important et un pronostic très sombre en phase métastatique, avec un taux de mortalité global de $25 \%$ à 10 ans. Son incidence annuelle est de 0,02 à 0,05 pour 100000 habitants (Meyer A et al.1999). Les tumeurs conjonctivales surviennent plus fréquemment chez les sujets de type caucasien que chez les sujets issus de races à la peau pigmentée, avec une médiane à 62 ans où l'incidence homme/femme est égale (Christine M. (2011).

Nous rapportons le cas d'une femme de 45 ans qui a développé un volumineux mélanome multi-récidivant de l'œil droit, chez qui nous avons pratiqué une exantération partielle a visée palliative.

\section{Observation}

Mme R.B âgée de 45 ans était reçue en consultation pour une tâche marron foncé sur la surface conjonctivale inféro-temporale de son œil droit évoluant depuis 20 ans. Cette tache de la taille d'un grain de riz était restée longtemps stationnaire, puis s'étalait lentement sur le plan horizontal vers la cornée. A sa première consultation dans le service d'ophtalmologie 10 ans auparavant, l'examen a révélé une acuité visuelle conservée à 10/10 aux deux yeux, et la présence d'un naevus sur la jonction limbo-sclérale temporale de l'œil droit d'environ 2 millimètres de diamètre. A cette période elle subissait une exérèse simple de la lésion. Deux ans après réapparaissait une tache semblable au même site qui fut elle aussi exérée dans un centre de santé. Trois ans après cette deuxième exérèse réapparaissait une masse bourgeonnante, noire, rapidement progressive qui envahissa en trois mois totalement l'œil droit avec une perte totale de la vision. A cet stade la patiente a subit une énucléation dont l'examen anatomopathologique révélait un mélanome superficiel. Après cette intervention la patiente était perdue de vue. Dix huit (18) mois plu tard, elle revint avec une masse bourgeonnante extra-oculaire ulceronecrotique, kystique et hémorragique saignant au moindre contact (Figure 1). Devant cette progression tumorale et l'altération de l'état général de la patiente, un bilan fut fait (NFS, Groupage sang et facteur Rhésus) avant une exanteration partielle et palliative de l'œil droit. La masse tumorale ainsi reséquée pesait $350 \mathrm{~g}$ et mesurait $6 \times 12 \times 5 \mathrm{~cm}$ (figure 2). L'examen anatomopathologique de la pièce révélait une prolifération 
tumorale étalée asymétrique et mal limitée intra épidermique latérale de type pagetoide; cette prolifération essentiellement faite de mélanocytes atypiques finement tatoués de mélanine mêlée à des vastes foyers de nécrose hémorragique était évocateur d'un mélanome (Figure 3). Une semaine après l'intervention la patiente a été soumise à une cure de chimiothérapie avec la cysplatine à la dose de $100 \mathrm{mg} / \mathrm{m}^{2}$ associée au 5-fluoro-Uracile à la dose de $1000 \mathrm{mg} / \mathrm{m}^{2}$. Le scanner effectué a montré une métastase cérébrale (Figure 4), il y a atteinte des ganglions lymphatiques sentinelles, sans localisation pulmonaire et abdomino-pelvienne. Un an après l'exanteration, l'état de la patiente était resté stable (figure 5).

Figure 1: tumeur avant exérèse (Aspect nécrotique et hémorragique)

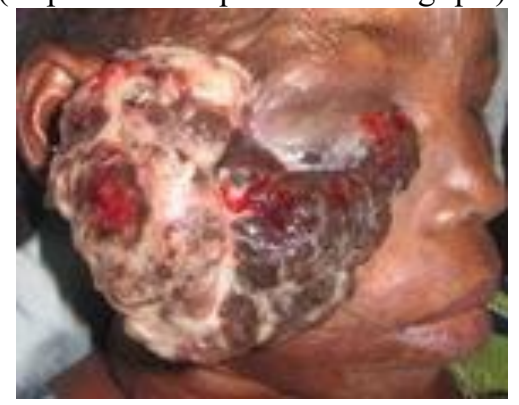

Figure 2: tumeur après exérèse

Figure 3(a et b): coupes histologiques de la tumeur

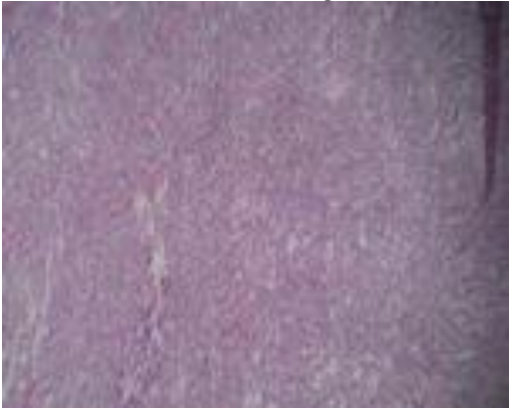

Figure 4: métastase cérébrale

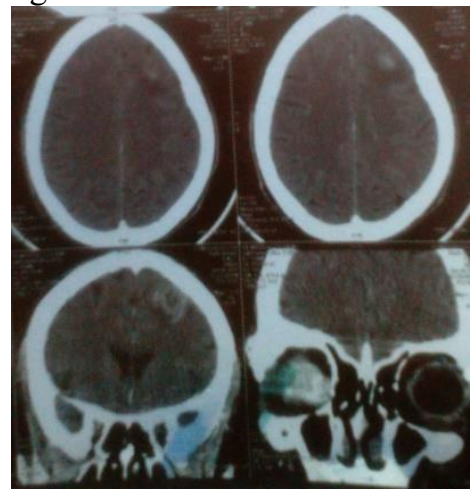
$(350 \mathrm{~g}$ et $6 \times 12 \times 5 \mathrm{~cm})$
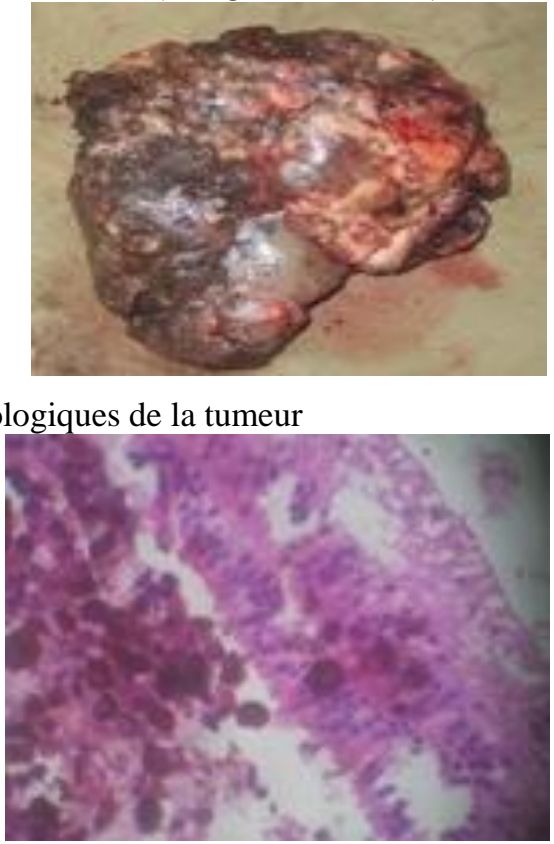

Figure 5: un an après exentération

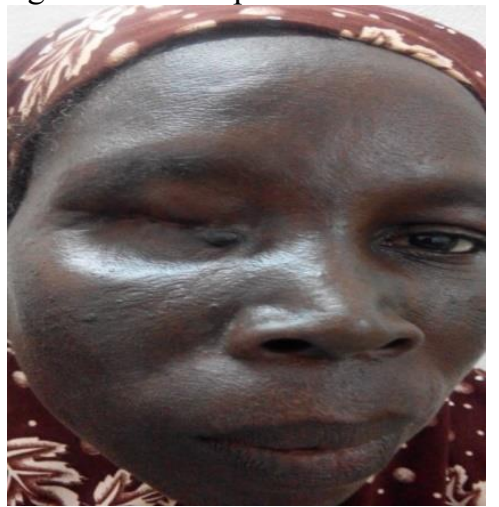




\section{Commentaires et Discussion}

Le mélanome peut rester longtemps asymptomatique ou être découvert incidemment pendant un contrôle ophtalmologique de routine. Il atteint d'une manière générale autant les hommes que les femmes de tous les âges, avec un pic d'incidence entre 40 et 50 ans. Desjardins et al (1999) ont retrouvé un âge moyen de 56 ans. Selon des données de la littérature 60 à $75 \%$ des mélanomes conjonctivaux se développent dans la mélanose primitive acquise (PAM), 20 à $25 \%$ dans des naevi conjonctivaux préexistants (naevi de jonction ou composés) et 10 à $25 \%$ se développent de novo dans l'épithélium conjonctival d'apparence normale (Solares et al 2000). Dans plus de $90 \%$ ils sont de nature melanotique, mais certains peuvent se présenter sous la forme de tumeurs amélanotiques (McGowan.H.D. (2004). et Carol S (2000). Des mélanomes nodulaires ayant survenu sur PAM ont été rapportées (Carol S et al 2011, Melichar B et al. 2008, et par Chazalon et al en 2007). Chez notre patiente le point de départ était le naevus de spitz. Le traitement initial des mélanomes conjonctivaux non métastatique dans la littérature est l'exérèse tumorale complète accompagnée d'une radiothérapie ou d'une prontothérapie (Carol 2000, Chazalon 2007) et plus récemment d'une chimiothérapie topique (mitomycine $\mathrm{C}$ en collyre). Dans notre cas clinique l'exérèse a été le premier geste chirurgical mais la carence de notre plateau technique en radiothérapie et en prontothérapie a fait que notre patiente n'a pas pu bénéficier d'un accompagnement thérapeutique dans ce sens. Suites aux récidives multiples et au volume de sa tumeur notre patiente a subit comme beaucoup de cas décrits (Desjardins 1999, Mc-Gowan.H.D. 2004, Duchateau 2005) une exentération bien que partielle de sa tumeur. 20 des 150 patients de Carol 2011 ont subit une exanteration et selon Kaplan-Meier 8\% des cas de mélanomes conjonctivaux subissent une exentération dans les 5 ans suivant le diagnostic.

En général, plus le cancer est antérieur au niveau de l'œil, plus les symptômes tardent à apparaître cliniquement, et les récidives sont fréquentes. Notre patiente a eu 4 récidives, dans l'étude de Moorfields et al. Le taux de récidive était de $39 \%$ (soit 22 patients, parmi ceux-ci 10 ont présenté une deuxième récidive, 5 une troisième, 3 une quatrième, 2 une cinquième, 1 une sixième et 1 une septième. Le délai de récidive varie entre 3 et 96 mois avec un délai moyen de 56 mois à 5 ans (McGowan.H.D.2004); un taux identique a été retrouvé par Desjardins et al. (1999) parmi eux 17,85\% avaient une multi-récidive, Ce taux était de $26 \%$ à 5 ans, de $51 \%$ à 10 ans et de $65 \%$ à 15 ans dans l'étude de Carol et al. (2011) en Philadelphie. Chez notre patiente la tumeur mesurait environ $2 \mathrm{~mm}$ de diamètre et était située à distance du limbe sclero-cornéen sur la conjonctive bulbaire du coté temporal de l'œil droit; les patients de Carol et 
al. (2011) avaient une localisation sur la conjonctive bulbaire dans 92\% et $63 \%$ étaient sur le quadrant temporal avec une taille tumorale en moyenne de $8 \mathrm{~mm}$ de diamètre.

Le mélanome est un des cancers pour lequel la survie à cinq ans est la plus élevée, elle est estimée entre 80 et $90 \%$. Mais cela est essentiellement lié aux mélanomes diagnostiqués à des stades précoces de la maladie, la survie chute à $70-80 \%$ à 10 ans (Melichar $\mathrm{B}$ et al. (2008). En revanche, au stade métastatique, le pronostic reste beaucoup plus sombre (Christine M. (2011). Le pronostic est meilleur pour les tumeurs du limbe et de la conjonctive bulbaire par rapport aux tumeurs du cul-de-sac et de la caroncule ; ceci a été mis en évidence par les études de Paridaens, de Fuchs et celle de Wollf-Rowendal. De même il est admis que le pronostic est meilleur pour les tumeurs développées sur mélanose ou sur des naevi par rapport à ceux survenant de novo (Chazalon et al 2007). La manifestation précoce des métastases est rare, Desjardins et al (1999) ont retrouvé un seul cas avec métastase sur 56 au diagnostic. Esmaeli et al ont observés des métastases à distance entre 3 et 15 ans après le diagnostic, Dans une série rétrospective sur 150 cas de mélanome conjonctival, Carol et al (2000) ont trouvés une prévalence des métastases de $16 \%$ à 5 ans, mais de $32 \%$ à 15 ans. La distribution des métastases des mélanomes conjonctivaux est dite similaire à celle des mélanomes cutanés (Missoten et al 2005).

\section{Conclusion}

Le mélanome de la conjonctive reste une tumeur grave de traitement difficile. Une forte suspicion clinique est indispensable au moment d'examens de lésions pigmentées de la conjonctive. Des traitements de pointe telle que la prontothérapie et la chimiothérapie topique permettent de nos jours une meilleure prise en charge et un meilleur pronostic de ces tumeurs rares.

\section{References:}

Desjardins L, Poncet P, Levy C, Schleinger P, Asselain B, Validire P. (1999). Facteurs pronostiques du mélanome malin de la conjonctive, étude anatomo-clinique sur 56 patients, J Fr. Ophthalmol; 22,3, 315-321.

Meyer A et al. (1999). Mélanome malin sur mélanose conjonctivale acquise primitive évoluant en mélanome malin, J Fr Ophthalmol; 22, 9,983-989.

Christine M. (2011). Le mélanome; Bulletin Infirmier du Cancer, 1, 11,1.

Solares-Zamoru J. (2000). Mélanose conjonctivale: évolution en mélanome malin; J Fr Ophthalmol, 23, 5, 525.

Mc-Gowan.H.D. (2004). Le mélanome malin de la conjonctive; Ophtalmologie - Conférences scientifiques, 2, 6 . 
Carol S.L, Jerry S.A, Kaan G, Jacqueline C, Gary M.V; Nicole G et al. (2000). Conjunctival melanoma: risk factors for recurrence, exanteration, metastasis, and death in 150 consecutive patients; Arch Ophthalmol, 118, 11:1497-1507.

Carol S.L, Jeremy M.S, Irina B, Hal S, Nina S.G, Sara L.E, Arman M et al. (2011). Conjunctival melanoma: outcomes based on tumor origin in 382 consecutive cases; Ophthalmology, 389-395.e2.

Melichar $\mathrm{B}$ et al. (2008). Mélanome malin de la conjonctive associée à deux autres lésions malignes primitives. J Fr Ophthalmol, 31, 4.

Duchateau N, Meyer A, Hugol D, Allali J, Audouin J, Renard G et al. (2005). Mélanome nodulaire sur mélanose acquise primitive de la conjonctive: À propos d'une observation anatomo-clinique; J Fr Ophtalmol; 2005; 28, 3, 331-335.

Chazalon E.P, Roux L, Patte J.H, Pommier S, Bonnet D, Meyer F. (2007). Mélanome conjonctival limbique développé sur mélanose acquise primitive; J Fr Ophtalmol, 10, 30, 8, 842.e1-842.e6.

Esmaeli B, Wang X, Youssef A, Gershenwald J.E. (2001). Patterns of regional and distant metastasis in patients with conjunctival melanoma. Ophthalmology, 108, 2, 101-5.

Missoten G.S, Keijser S, Wolff - Roosendaal D. (2005). Conjunctival melanoma in Netherlands: a nationwide study. Invest Ophthalmol Vis Sci, 46: $75-82$. 